\title{
Trauma to the Pelvis: Injuries to the Rectum and Genitourinary Organs
}

\author{
Karen Wu • Joseph A. Posluszny Jr. • Jeffrey Branch • \\ Elizabeth Dray • Robert Blackwell • Jessica Hannick • \\ Fred A. Luchette
}

Published online: 10 January 2015

(C) Springer International Publishing AG 2015

\begin{abstract}
Pelvic trauma is associated with high mortality rates. Blunt pelvic injuries from high-energy mechanisms are often associated with pelvic fractures and injuries to the rectum and genitourinary (GU) tract. In addition, due to close anatomic proximity, penetrating pelvic trauma can injure the bony pelvis, rectum, and GU tract concomitantly. As a result, the assessment and management of pelvic trauma requires a multifaceted approach involving orthopedics, trauma surgery and urology.
\end{abstract}

Keywords Pelvic trauma $\cdot$ Pelvic fracture $\cdot$ Rectal trauma Bladder injury $\cdot$ Ureteral injury $\cdot$ Urethral injury

This article is part of the Topical Collection on Trauma to the Pelvis

$\mathrm{K}$. Wu $\cdot$ J. A. Posluszny Jr. J. Branch $\cdot$ F. A. Luchette

Edward Hines, Jr. Veterans Administration Medical Center, Stritch

School of Medicine, Loyola University of Chicago, Hines, IL 60141, USA

K. Wu

Department of Orthopaedic Surgery, Stritch School of Medicine, Loyola University of Chicago, Maywood, IL 60153, USA

J. A. Posluszny Jr.

Department of Surgery, Stritch School of Medicine, Loyola University of Chicago, 2160 South First Avenue, Maywood, IL 60153, USA

J. Branch $\cdot$ E. Dray $\cdot$ R. Blackwell $\cdot$ J. Hannick

Department of Urology, Stritch School of Medicine, Loyola

University of Chicago, 2160 South First Avenue, Maywood,

IL 60153, USA

F. A. Luchette $(\square)$

Department of Surgery, Stritch School of Medicine, Loyola

University of Chicago, Hines, IL 60141, USA

e-mail: Frederick.Luchette@va.gov

\section{Introduction}

Blunt trauma frequently results in a pelvic injury with a frequency of $9 \%$. These wounds can range from clinically insignificant minor pelvic fractures to life-threatening injuries that produce exsanguination ( $0.5 \%$ of all blunt trauma patients). The overall mortality rate of patients with a disruption of the pelvic ring is approximately $6 \%$. Uncontrolled pelvic hemorrhage after blunt trauma accounts for $39 \%$ of related deaths. Anterior-posterior compression and vertical shear injuries are associated with a higher incidence of pelvic vascular injury and hemorrhage compared to lateral impact fractures. Blunt pelvic injuries are often associated with pelvic fractures and injuries to the rectum and genitourinary (GU) tract. In addition, due to close anatomic proximity, penetrating pelvic trauma can injure the bony pelvis, rectum, and GU tract concurrently.

The literature contains numerous methods of management resulting in a lack of consensus for a universally accepted guideline. However, the recent evolution of rapid pelvic stabilization by external fixation or pelvic binding and of bleeding control by angiographic embolization or preperitoneal pelvic packing has significantly decreased the mortality rates of severe pelvic fractures. A multidisciplinary approach is crucial to optimal patient outcome. No single specialty has all the skills or controls all the resources that may be necessary. Emergency medicine physicians, trauma and critical care surgeons, orthopedic surgeons, urologists, and interventional radiologists should play protagonist roles in a well-orchestrated trauma team that manages these complex patients. This manuscript will review the current state of management for the specific injuries associated with pelvic injuries.

\section{Pelvic Ring Fractures}

The initial assessment and resuscitation of a high-energy trauma patient should follow an Advanced Trauma Life Support 
protocol. While most ( $80 \%$ ) genitourinary and rectal injuries from blunt pelvic force are associated with pelvic ring disruption [1], only a small subset of all pelvic fractures are associated with genitourinary or rectal injury (2-25\%) [2-4]. The orthopedic surgeon has a critical role in the early assessment and management of the bony injury and must play an integral role assisting to identify those suspected with associated injuries $[5,6,7 \bullet \bullet, 8,9]$.

For the hemodynamically unstable patient with an unstable external rotation pelvic ring injury (APC and VS), a pelvic binder or sheet will assist with temporary stabilization and it can be performed quickly in the trauma bay. The ease and rapidity of application of either as well as its efficacy have replaced the emergent use of the C-clamp or an external fixator [10] with improved alignment after placement of a pelvic binder. However, when used with a LC fracture, the deformity will be worsened [11]. The sheet or binder should be placed directly over the greater trochanters to be most effective $[12,13]$. It is not clear how long the binders can be left in place [14]. Care must be taken to prevent skin necrosis from developing beneath the binder.

Continuous assessment of the patient's hemodynamics is necessary. If a patient remains hypotensive or if only a transient responded to fluid and/or blood product resuscitation, pelvic angioembolization and pelvic external fixation with preperitoneal pelvic packing (EF-PPP) are two options for hemorrhage control. If the patient requires emergent laparotomy for intra-abdominal injury, EF-PPP is most easily performed. With EF-PPP, pelvic external fixation is performed to stabilize the pelvis, keeping access clear to the abdomen and pelvis. Laparotomy with preperitoneal packing controls the hemorrhage.

If emergent laparotomy is not indicated and intravenous contrast extravasation is seen on pelvic CT scan, then proceeding for emergent angiography and embolization is the next step in management [15]. Miller et al. showed that contrast blush on pelvic CT had sensitivity of $60 \%$ and specificity of $92 \%$ in predicting bleeding on angiography [16]. A more recent study using modern 64-slice multidetector CT showed improved sensitivity of $82 \%$ and specificity of $95 \%$ [17]. After embolization, if the patient remains hemodynamically unstable after, consideration should be given to repeat angiography or supplementary EF-PPP.

Both angioembolization and PPP via laparotomy have been found to be equally effective in small retrospective studies [18, 19]. Cheng et al. in a retrospective study concluded that survival was improved when managed with PPP versus angiography [20]. Some centers have shifted from angiography to PPP as the initial intervention to control hemorrhage $[21,22 \cdot$, 23]. A recently published guideline on the management of hemodynamically unstable pelvic trauma also recommends PPP as the initial management for pelvic hemorrhage [24]. However, other trauma centers prefer angiography [25]. The US Eastern Association for the Surgery of Trauma (EAST) update on
Management Guidelines on Hemorrhage from Pelvic Trauma does not recommend one approach over the other [10].

The reluctance to utilize PPP is due to several issues. First, many surgeons, especially in the USA, are unfamiliar with the technique. Another concern is performing an open surgical procedure in a hemodynamically unstable and possibly coagulopathic patient. Additionally, the laparotomy packs used have been shown to become colonized with bacteria, although there has been no evidence to suggest that there is associated increase in infection rate [26]. Finally, the patient will need to return to the OR for pack removal.

The barriers to angiography are the ability to manage an unstable patient in the angiography suite, time and resources required for endovascular intervention [27], and concern for skin ischemia/necrosis in the gluteal area which may compromise a surgical incision-needed definitive fracture fixation [28-30].

Angioembolization and EF-PPP should be viewed as complementary rather than competitive modalities [31]. Additional studies are needed to evaluate if one technique is advantageous over the other. Institution-specific protocols and outcomes will also be affected by the expertise of local providers and availability of resources.

Open pelvic fractures have high mortality rates and are often associated with urogenital and intra-abdominal injuries [32]. Careful initial inspection of the perineum for open wounds should be performed in the trauma bay to avoid missing the diagnosis. When associated with rectal laceration, mortality is especially high [33]. For open pelvic fractures, wound packing for hemorrhage control, early diverting colostomy, external fixation, and aggressive serial debridement should be performed [34].

\section{Penetrating Trauma to the Pelvic Ring}

Penetrating pelvic injuries are a separate subset of pelvic trauma where pelvic fracture may be associated with injury to the genitourinary system and rectum. As with blunt pelvic injuries, initial assessment and resuscitation should follow the principles of ATLS. Firearm wounds to the pelvic region result in fractures and injuries to the bowel, urinary system, and vasculature. When there is a concomitant bowel injury, the risk for infection is significant. If the hip joint is involved or the pelvic fracture will require fixation, then urgent irrigation and debridement of the joint/fracture is indicated in conjunction with empiric antibiotics. Definitive fracture fixation should be delayed until the risk of infection is minimized. When the fracture does not require operative fixation, empiric antibiotics may be administered [35].

\section{Definitive Treatment of Pelvic Fractures}

A comprehensive discussion on the treatment of pelvic fractures is covered in another article in this issue. The following 
is a brief summary of definitive orthopedic treatment for the pelvic ring injuries associated with genitourinary and rectal injuries. After resuscitation, imaging of the pelvis should be obtained. Five views of the pelvis (anterior/posterior, inlet, outlet, and Judet views) and thin-sliced CT scan will assist with planning definitive surgical fixation. Management of concomitant genitourinary and/or rectal injuries should be planned jointly with the orthopedic surgeon.

External fixation can be used as definitive stabilization of the anterior pelvic ring. If an extraperitoneal bladder rupture is present or suprapubic catheter is placed, consideration for definitive treatment with external fixation rather than internal plating may be considered due to the contamination of the retropubic space and concern for infection. Similarly, in cases with bowel contamination, open fracture and other situations where risk of infection is high, external fixation has been shown to be associated with reduced rates of osteomyelitis. When external fixation is used for definitive fixation, the construct is often revised to add or switch from iliac crest pins to supra-acetabular pins.

For open reduction and internal fixation of the pubic symphysis and anterior ring, a Pfannenstiel incision is preferred, which also provides adequate exposure for the urologist to address a bladder injury. For morbidly obese patients where open surgery and anterior plating are problematic [36], the InFix system was developed to manage the wound healing problems and biomechanical failure from increased forces seen with open anterior plating [37].

If indicated, posterior pelvic stabilization can be achieved via percutaneous sacroiliac screw fixation or open reduction and internal fixation depending on the fracture pattern requirements and consideration for concomitant visceral injuries and risk of infection.

\section{Injuries to the Rectum}

Most commonly, injuries to the rectum occur with penetrating rather than blunt pelvic trauma. However, with significant pelvic fractures, injury to the rectum may occur. In this section, the mechanisms of injury and injury patterns associated with rectal trauma, methods for evaluating for rectal injury, and the myriad of options for managing rectal injury will be reviewed. It should be noted that much of the literature combines the management of colon and rectal injuries. This section will focus solely on extraperitoneal wounds of the rectum. More proximal injuries are managed as a colon or intraperitoneal bowel injury.

Traditionally, rectal trauma had been managed with the principle of the four Ds: divert, drain, direct repair, and distal washout [38]. These management principles were developed during the Vietnam War and demonstrated improved survival when compared to prior wartime management. Challenging these current principles is difficult as extraperitoneal rectal injuries are rare, limiting a large-scale study, and the clinical consequences of pelvic sepsis without proximal diversion of the fecal stream can be a disastrous scenario in an often already multiply injured patient. In addition, extrapolating the injury patterns and management of high-velocity penetrating pelvic injuries in combat (36\% 1-week mortality for combined vascular and rectal pelvic trauma) [39] to the civilian setting is impractical.

A scale for rectal injuries has been developed by the American Association for the Surgery of Trauma. While this scale is useful for coding and research purposes, it does not take into account the anatomic location (relationship to peritoneum) of the injury, mechanism of injury or associated pelvis, or intraabdominal injuries which limits its clinical usefulness.

\section{Associated Injuries}

As this chapter clearly demonstrates, penetrating wounds to the pelvis often involve the rectum, GU organs, and bony pelvis concurrently. A review of 22 years of penetrating bladder injuries in Brazil showed that $41 \%$ of patients also had a rectal injury [40]. Penetrating rectal injuries themselves are associated with a $39-47 \%$ incidence of GU injury with the bladder being most frequently injured [41]. Although less common with blunt bony pelvic injury, $25 \%$ of patients with an open pelvic fracture have an associated rectal laceration (Jones-Powell class III). In this study, the highest mortality and highest ISS scores were for patients with a combination of open pelvic fracture and rectal laceration underscoring the synergistic effect of combined pelvic injuries on mortality [42]. Aside from the structures confined to the pelvis, the small bowel occupies the intraperitoneal portion of the pelvis and is also injured in $36 \%$ of penetrating wounds to the rectum [41]. In addition, other intra-abdominal organs or vascular structures may be injured as determined by the trajectory of the missile or stab wound.

\section{Suspicion of Injury}

Typically, any penetrating injury to the pelvis or significant pelvic fracture should raise concern for a rectal injury. As mentioned previously, rectal trauma requiring surgical intervention is rare with a blunt trauma. In contrast, when a penetrating injury traverses the pelvis, the rectum must be evaluated. We recommend direct visualization of the rectum with either rigid proctoscopy or flexible sigmoidoscopy [43]. During both procedures, the rectal mucosa is visualized circumferentially and assessed for injury. If injury is present, repair will then depend on the degree of injury (hematoma v. laceration), anatomic location (intra- or extraperitoneal), and clinical scenario (hemodynamic status). Noninvasive imaging 
with $\mathrm{CT}$ of the rectum with rectal contrast should be used with caution as immediate imaging may not accurately assess highenergy wounds to the rectum that are only a partial injury to the wall that subsequently convert into a full thickness wound.

\section{Management of Injury}

The extraperitoneal rectum is not easily mobilized, and mobilization, resection, and anastomosis are difficult in the elective setting and even more so in trauma. Therefore, diversion of the fecal stream with a proximal colostomy with or without presacral drainage and primary repair is the current standard of care. Primary diversion regardless of the size of the rectal injury is the most prudent and conservative management option. In contrast, intraperitoneal rectal injuries should be treated in a similar fashion to left colon injuries with either primary repair or resection and anastomosis with or without diversion. This management is highlighted by a guideline for penetrating rectal injuries developed at the University of Tennessee at Memphis which based the type of repair on the anatomic location of injury. Rectal injuries in the proximal two thirds of the rectum could be managed with primary repair or resection and anastomosis with proximal diversion at the surgeon's discretion. Those wounds in the distal one third of the rectum could undergo primary repair if accessible transanally, with proximal diversion utilized if the injury was not easily accessible, with presacral drainage. Despite the use of the pathway, only three of 50 patients with an extraperitoneal rectal injury were managed without proximal diversion, underscoring the surgeons' preference for proximal diversion in the management of distal rectal injuries [41]. Data and reports challenging the need for proximal diversion are lacking. One prospective study, albeit a small study, evaluated the benefit of eliminating proximal diversion in the management of rectal injuries. However, the study only included patients with "nondestructive" ( $<25 \%$ lumen involvement) penetrating injuries [44]. No study thus far can reliably exclude proximal fecal diversion as standard of care for penetrating rectal injury.

Proximal diversion does not always require a laparotomy though. Navasaria et al. reported the successful management of 20 patients with penetrating rectal trauma who underwent diagnostic laparoscopy to assess for intraperitoneal violation and injury. When the injury was determined to be extraperitoneal, a loop colostomy was created [45].

Presacral drainage had previously been considered to be the standard of care based on management with the four Ds from the Vietnam War. The need for presacral drainage was based on a military combat rectal injury, and these injuries may require drainage, given the extent of tissue destruction. However, several retrospective studies in civilian trauma have shown no increase in pelvic sepsis or infection with the omission of presacral drainage when diverting colostomy is performed [46, 47]. Additionally, a prospectively randomized trial of 48 patients with penetrating extraperitoneal injuries managed with diverting colostomy and presacral drainage demonstrated no benefit for presacral drainage [48]. Therefore, in civilian-penetrating pelvic injuries with a rectal wound and without massive tissue destruction, presacral drainage is unnecessary.

Direct repair of the injury is not necessary either. A retrospective study of 30 consecutive patients with penetrating extraperitoneal rectal injuries who were all treated with diverting colostomy with or without direct repair and presacral drainage showed no infectious or survival benefit for direct repair or presacral drainage [49]. This data is supported by other retrospective studies in which direct repair was not performed at all following proximal diversion, and there was no evidence of sepsis or associated morbidity $[47,50]$.

The management of combined bladder and rectal injuries may require more than just proximal diversion, however. High rates $(24 \%)$ of rectovesical and rectourethral fistula are associated with combined GU and rectal injuries [51]. Therefore, in these instances, debridement of necrotic rectum with primary repair, proximal diversion, and the placement of an omental pedicle flap between the rectum and GU injury may reduce fistula formation [40] especially in cases of combined posterior bladder and anterior rectal injuries [52, 53].

Two to 3 months following the injury, the rectum and distal colon can be evaluated for healing and patency with a barium enema and, if adequate, the colostomy may be reversed with high rates of success.

In summary, rectal trauma occurs most commonly with penetrating injuries and is quite often associated with injuries of the bladder, distal urinary system, and small bowel. Any suspicion for rectal trauma should prompt visualization of the rectum with various endoscopic equipment. Currently, injuries to the rectum should be managed with proximal diversion with additional consideration for tissue debridement and omental flap placement when there is concomitant GU injury.

\section{Injuries to the Lower Genitourinary Tract}

When caring for the multiply injured trauma patient, each surgical discipline maintains expertise in the evaluation and management of their respective system. For GU trauma, the American Urological Association (AUA) has published the 2014 AUA urotrauma guideline. The guideline panel performed a systematic review of 372 articles covering urologic trauma, spanning the years 1990-2012. Organ-based guideline statements were issued for ureteral, bladder, and genital trauma and serve to guide surgeons in the appropriate methods of recognition, evaluation, and management of GU injuries $[54 \bullet \bullet$. 


\section{Ureteral Injury}

Ureteral injury represents the rarest form of GU trauma, resulting from high-speed blunt trauma, penetrating wounds, or iatrogenic injury. Injury from external trauma occurs in $4 \%$ of penetrating injuries and $1 \%$ of blunt trauma. These patients generally have concomitant injuries, frequently to the kidneys (10-28 \%), bladder (5\%), small bowel (39-65\%), and colon (28-33\%), with overall mortality approaching $33 \%$ [55]. Imaging should be obtained in (1) patients with penetrating trauma and hematuria or (2) blunt trauma and gross hematuria or (3) microscopic hematuria and hypotension [54••]. Appropriate imaging modalities include $\mathrm{CT}$ with contrast and delayed films. Thirty-three percent of ureteral pelvic junction disruptions, seen in rapid deceleration injuries, and $25 \%$ of overall ureteral injuries do not present with hematuria, however, so individuals with high-risk mechanisms of injury (i.e., rapid deceleration or nearby injuries) should undergo radiologic evaluation [56].

Management of ureteral injury relies on several fundamental operative principles: (1) ureteral mobilization with wide adventitial sparing to prevent devascularization; (2) debridement of damaged tissue with careful consideration for possible blast effect; (3) a spatulated, tension-free repair over a stent using fine, absorbable monofilament suture; (4) drain placement; and (5) retroperitonealization of the ureter following repair [59]. Lower ureteral injuries are managed with ureteroneocystostomy (ureteral reimplantation), which sometimes requires bladder mobilization or bladder flap. In minor contusions, ureteral stenting may be attempted as definitive management. With unstable patients, the ureter is ligated with nonabsorbable suture, and percutaneous nephrostomy tubes are placed until definitive repair [54••].

Delayed diagnosis of ureteral injury should be evaluated with retrograde pyelogram and stent placement. If unsuccessful, percutaneous nephrostomy and antegrade stent placement should be attempted with subsequent urologic referral.

\section{Bladder Injury}

As a result of its protected location within the true pelvis, the majority of bladder injuries are associated with pelvic fractures and concurrent bladder rupture is seen overall in 2$11 \%$ of cases [58]. Incidence increases to $29 \%$ when there is also gross hematuria $(\mathrm{GH})$. Injuries may also be the result of penetrating trauma or rupture secondary to a substantial compression force [54*0]. GH alone is very sensitive for bladder rupture, as it is present in $77-100 \%$ of injuries [54••].

Radiologic findings on CT scan that predict a higher risk of bladder injury include a pelvic fluid collection on CT, diastasis of the symphysis pubis greater than $1 \mathrm{~cm}$, obturator ring fracture with displacement greater than $1 \mathrm{~cm}$, a palpable bladder, abdominal distention, and/or rising serum creatinine/blood urine nitrogen, the latter being associated with a missed urinary tract injury [59]. Bladder injuries are classified as extraperitoneal (EP) versus intraperitoneal (IP), occurring in 60-86 and $14-30 \%$ of trauma patients, respectively [54••, $60 \bullet$.

When a bladder injury is suspected and Foley catheter has been placed, retrograde cystography should be performed with $300 \mathrm{~cm}^{3}$ contrast. Antegrade bladder filling following IV contrast administration inadequately distended the bladder for evaluation and may delay diagnosis [54••]. Either computed tomography or plain films (with an AP and lateral view) can be obtained after retrograde contrast infusion [58]. Finally, a post-void film is essential to document extravasation which may be obscured when the bladder is filled with contrast.

IP bladder rupture warrants emergent surgical repair, in the hemodynamically stable patient, given the risk for IP sepsis and inadequate healing with catheter drainage alone [54••]. Between 2002 and 2006, 8565 patients were identified to have experienced bladder injury in Deibert and Spencer's analysis of the National Trauma Data Bank [60•]. They reported a striking discrepancy between the perceived standard practice of surgical repair of IP bladder rupture (as recommended by the AUA urotrauma guideline) and a reported rate of repair of only $76 \%$.

When repairing an intraperitoneal bladder rupture, debridement to viable tissue is essential, followed by watertight closure in two layers with absorbable sutures. Permanent sutures in the urinary tract should be avoided to prevent stone formation and infection. Urethral catheter drainage is preferred over suprapubic catheters as urethral drainage is associated with decreased hospital stay and lower morbidity [54••].

In hemodynamically unstable patients with a bladder injury, urinary diversion and delayed urinary tract repair may be necessary [61]. For patients undergoing abbreviated laparotomy found to have bladder rupture, it is reasonable to place ureteral stents and externalize them to the skin to divert the urine. Alternatively, to collect urine in the patient whose abdomen will be left open, either a closed suction drain in the proximity of the bladder or an abdominal wound vacuum dressing has been used at our institution.

EP bladder ruptures can usually be managed conservatively with catheter drainage, although several indications exist for immediate repair. These include foreign bodies/bone fragments in the bladder, associated vaginal/rectal injury, laparotomy for other reasons, or if the patient is a candidate for open reduction internal fixation of an associated pelvic fracture [59]. A longitudinal cystotomy is performed to allow complete inspection and repair of bladder injuries. The ureteral orifices are examined for clear efflux, and the integrity of the bladder neck is assessed. Urethral catheter drainage after bladder repair is favored.

Patients with an EP bladder rupture managed nonoperatively should have a follow-up cystogram performed 
to rule out persistent extravasation which can be present in $15 \%$ of cases [59]. Mortality rates attributed to traumatic bladder injury are estimated nationally at $10.8 \%$ for EP and $6.5 \%$ for IP ruptures, respectively [60 $\left[0^{\bullet}\right.$.

\section{Urethral Injury}

Urethral injuries are categorized as posterior (from the bladder neck to the membranous urethra) or anterior (from the bulbar urethra to the meatus). Posterior urethral injuries generally result from blunt trauma and can occur concurrently with bladder injuries. They are also commonly associated with pelvic fractures (up to $10 \%$ ) as the posterior urethra is adherent to the pubic symphysis [58]. Blood at the meatus is the most common finding, though patients may exhibit an inability to void, a palpable bladder, a butterfly perineal hematoma, or a high-riding prostate. They may also be found upon difficult Foley catheter placement [59].

If posterior urethral injury is suspected, a retrograde urethrogram (RUG) should be performed. If a catheter has been placed, a peri-catheter RUG should be obtained to confirm appropriate placement [54・•]. Partial urethral tears are best managed by gentle urethral catheter placement with confirmatory RUG. If a posterior urethral disruption is identified, immediate open reconstruction is rarely used due to the high complication rates [61]. Instead, either suprapubic catheter with delayed reconstruction or primary antegrade or retrograde endoscopic realignment over a catheter is preferred.

Anterior urethral injuries generally occur during a straddle injury and involve the bulbar urethra. Penile urethral injury is rare and may result from penetrating injuries to the penis. Signs include presence of blood at the urethral meatus, perineal hematoma, and urinary retention. RUG is recommended in the setting of signs or a suspicious mechanism of injury. If a minor or incomplete injury is seen, primary realignment over a urethral catheter is the standard of care. Primary surgical repair is recommended for lowvelocity gunshot wounds or stab wounds [54・•]. Debridement can be minimized as the robust blood supply to the sponge often results in spontaneous healing. Suprapubic cystotomy and delayed repair should be utilized in the event of a high-velocity gunshot wound or severe straddle injury.

Urethral injury may also occur in women in up to $6 \%$ of pelvic fractures [57]. Suspicious findings on genital exam include vulvar edema or blood at the vaginal introitus. Diagnosis is confirmed by EUA and urethroscopy. Female urethral injuries should be immediately repaired or realigned over a catheter, if feasible, to prevent urethral obliteration [57]. Any concomitant vaginal lacerations must be carefully closed to prevent fistula formation.

\section{Conclusions}

This chapter details the complex and multidisciplinary management of pelvic trauma. The need for prompt communication and recognition of injuries outside of your own specialty will help in the overall management of these patients. Ensuring quick pelvic ring stabilization with a mind toward hemorrhage control initially with pelvic binding and then with either angioembolization and/or packing, early recognition and diverting of the fecal stream for penetrating rectal injuries and management of lower GU injuries in line with the latest AUA guidelines are a key to the successful treatment of patients with pelvic trauma.

\section{Compliance with Ethics Guidelines}

Conflict of Interest Karen Wu, Joseph A. Posluszny Jr., Jeffery Branch, Elizabeth Dray, Robert Blackwell, Jessica Hannick, and Fred A. Luchette declare that they have no conflicts of interest.

Human and Animal Rights and Informed Consent This article does not contain any studies with human or animal subjects performed by any of the authors.

\section{References}

Papers of particular interest, published recently, have been highlighted as:

- Of importance

•• Of major importance

1. Matlock KA et al. Blunt traumatic bladder rupture: a 10-year perspective. Am Surg. 2013;79(6):589-93.

2. Durrant JJ et al. Pelvic fracture-related urethral and bladder injury. J R Army Med Corps. 2013;159 Suppl 1:i32-9.

3. Aihara $\mathrm{R}$ et al. Fracture locations influence the likelihood of rectal and lower urinary tract injuries in patients sustaining pelvic fractures. J Trauma. 2002;52(2):205-8. discussion 208-9.

4. Watnik NF, Coburn M, Goldberger M. Urologic injuries in pelvic ring disruptions. Clin Orthop Relat Res. 1996;329:37-45.

5. Young JW et al. Pelvic fractures: value of plain radiography in early assessment and management. Radiology. 1986;160(2):445-51.

6. Dalal SA et al. Pelvic fracture in multiple trauma: classification by mechanism is key to pattern of organ injury, resuscitative requirements, and outcome. J Trauma. 1989;29(7):981-1000. discussion 1000-2.

7.• Marsh JL et al. Fracture and dislocation classification compendium-2007: Orthopaedic Trauma Association classification, database and outcomes committee. J Orthop Trauma. 2007;21(10 Suppl):S1-133. Large database outcomes for traumatic pelvic fracture.

8. Ter-Grigorian AA, Kasyan GR, Pushkar DY. Urogenital disorders after pelvic ring injuries. Cent Eur J Urol. 2013;66(3):352-6.

9. Osterhoff $\mathrm{G}$ et al. Comparing the predictive value of the pelvic ring injury classification systems by Tile and by Young and Burgess. Injury. 2014;45(4):742-7.

10. Cullinane DC et al. Eastern Association for the Surgery of Trauma practice management guidelines for hemorrhage in pelvic 
fracture - update and systematic review. J Trauma. 2011;71(6): $1850-68$.

11. Toth L et al. Efficacy and safety of emergency non-invasive pelvic ring stabilisation. Injury. 2012;43(8):1330-4.

12. Bonner TJ et al. Accurate placement of a pelvic binder improves reduction of unstable fractures of the pelvic ring. J Bone Joint Surg (Br). 2011;93(11):1524-8.

13. Prasarn ML et al. Does application position of the T-POD affect stability of pelvic fractures? J Orthop Trauma. 2013;27(5):262-6.

14. Knops SP et al. Randomised clinical trial comparing pressure characteristics of pelvic circumferential compression devices in healthy volunteers. Injury. 2011;42(10):1020-6.

15. Bozeman $\mathrm{MC}$ et al. Use of computed tomography findings and contrast extravasation in predicting the need for embolization with pelvic fractures. Am Surg. 2012;78(8):825-30.

16. Miller PR et al. External fixation or arteriogram in bleeding pelvic fracture: initial therapy guided by markers of arterial hemorrhage. J Trauma. 2003;54(3):437-43.

17. Mohseni $\mathrm{S}$ et al. The diagnostic accuracy of 64-slice computed tomography in detecting clinically significant arterial bleeding after pelvic fractures. Am Surg. 2011;77(9):1176-82.

18. Tai DK et al. Retroperitoneal pelvic packing in the management of hemodynamically unstable pelvic fractures: a level I trauma center experience. J Trauma. 2011;71(4):E79-86.

19. Osborn PM et al. Direct retroperitoneal pelvic packing versus pelvic angiography: a comparison of two management protocols for haemodynamically unstable pelvic fractures. Injury. 2009;40(1):54-60.

20. Cheng, M., et al., Improvement in institutional protocols leads to decreased mortality in patients with haemodynamically unstable pelvic fractures. Emerg Med J, 2013.

21. Burlew $\mathrm{CC}$ et al. Preperitoneal pelvic packing/external fixation with secondary angioembolization: optimal care for life-threatening hemorrhage from unstable pelvic fractures. J Am Coll Surg. 2011;212(4):628-35. discussion 635-7.

22. Cothren CC et al. Preperitonal pelvic packing for hemodynamically unstable pelvic fractures: a paradigm shift. J Trauma. 2007;62(4): 834-9. discussion 839-42. Description of preperitoneal packing.

23. Marzi I, Lustenberger T. Management of bleeding pelvic fractures. Scand J Surg. 2014;103(2):104-11.

24. Magnone $\mathrm{S}$ et al. Management of hemodynamically unstable pelvic trauma: results of the first Italian consensus conference (cooperative guidelines of the Italian Society of Surgery, the Italian Association of Hospital Surgeons, the Multi-specialist Italian Society of Young Surgeons, the Italian Society of Emergency Surgery and Trauma, the Italian Society of Anesthesia, Analgesia, Resuscitation and Intensive Care, the Italian Society of Orthopaedics and Traumatology, the Italian Society of Emergency Medicine, the Italian Society of Medical Radiology-Section of Vascular and Interventional Radiology - and the World Society of Emergency Surgery). World J Emerg Surg. 2014;9(1):18.

25. Abrassart S, Stern R, Peter R. Unstable pelvic ring injury with hemodynamic instability: what seems the best procedure choice and sequence in the initial management? Orthop Traumatol Surg Res. 2013;99(2):175-82.

26. Granchi TS et al. Patterns of microbiology in intra-abdominal packing for trauma. J Trauma. 2004;56(1):45-51.

27. Schwartz DA et al. Are we delivering two standards of care for pelvic trauma? Availability of angioembolization after hours and on weekends increases time to therapeutic intervention. J Trauma Acute Care Surg. 2014;76(1):134-9.

28. Gilleard O, Stammers J, Ali F. Gluteal necrosis following pelvic fracture and bilateral internal iliac embolization: reconstruction using a transposition flap based on the lumbar artery perforators. Int J Surg Case Rep. 2012;3(2):86-8.
29. Takahira $\mathrm{N}$ et al. Gluteal muscle necrosis following transcatheter angiographic embolisation for retroperitoneal haemorrhage associated with pelvic fracture. Injury. 2001;32(1):27-32.

30. Matityahu A et al. Acute complications of patients with pelvic fractures after pelvic angiographic embolization. Clin Orthop Relat Res. 2013;471(9):2906-11.

31. Suzuki T, Smith WR, Moore EE. Pelvic packing or angiography: competitive or complementary? Injury. 2009;40(4):343-53.

32. Dong JL, Zhou DS. Management and outcome of open pelvic fractures: a retrospective study of 41 cases. Injury. 2011;42(10):1003-7.

33. Cannada LK et al. The Jones-Powell classification of open pelvic fractures: a multicenter study evaluating mortality rates. J Trauma Care Surg. 2013;74(3):901-6.

34. Govaert $\mathrm{G}$ et al. Prevention of pelvic sepsis in major open pelviperineal injury. Injury. 2012;43(4):533-6.

35. Miller AN, Carroll EA, Pilson HT. Transabdominal gunshot wounds of the hip and pelvis. J Am Acad Orthop Surg. 2013;21(5):286-92.

36. Sems SA et al. Elevated body mass index increases early complications of surgical treatment of pelvic ring injuries. J Orthop Trauma. 2010;24(5):309-14.

37. Vaidya $\mathrm{R}$ et al. Treatment of unstable pelvic ring injuries with an internal anterior fixator and posterior fixation: initial clinical series. J Orthop Trauma. 2012;26(1):1-8.

38. Lavenson GS, Cohen A. Management of rectal injuries. Am J Surg. 1971;122(2):226-30.

39. Arthurs Z, Kjorstad R, Mullenix P, Rush Jr RM, Sebesta J, Beekley A. The use of damage-control principles for penetrating pelvic battlefield trauma. Am J Surg. 2006;191(5):604-9.

40. Pereira BM, Reis LO, Calderan TR, de Campos CC, Fraga GP. Penetrating bladder trauma: a high risk factor for associated rectal injury. Adv Urol. 2014;2014:386280.

41. Weinberg JA, Fabian TC, Magnotti LJ, Minard G, Bee TK, Edwards $\mathrm{N}$, et al. Penetrating rectal trauma: management by anatomic distinction improves outcome. J Trauma. 2006;60(3):508-13.

42. Cannada LK, Taylor RM, Reddix R, Mullis B, Moghadamian E, Erickson M, et al. The Jones-Powell Classification of open pelvic fractures: a multicenter study evaluating mortality rates. J Trauma Acute Care Surg. 2013;74(3):901-6.

43. Ross GL, Dodd O, Lipham JC, Campbell JK. Rectal perforation in unstable pelvic fractures: the use of flexible sigmoidoscopy. Injury. 2001;32(1):67-8.

44. Gonzalez RP, Phelan 3rd H, Hassan M, Ellis CN, Rodning CB. Is fecal diversion necessary for nondestructive penetrating extraperitoneal rectal injuries? J Trauma. 2006;61(4):815-9.

45. Navsaria PH, Shaw JM, Zellweger R, Nicol AJ, Kahn D. Diagnostic laparoscopy and diverting sigmoid loop colostomy in the management of civilian extraperitoneal rectal gunshot injuries. Br J Surg. 2004;91(4):460-4.

46. Steinig JP, Boyd CR. Presacral drainage in penetrating extraperitoneal rectal injuries: is it necessary? Am Surg. 1996;62(9):765-7.

47. Navsaria PH, Edu S, Nicol AJ. Civilian extraperitoneal rectal gunshot wounds: surgical management made simpler. World J Surg. 2007;31(6):1345-51.

48. Gonzalez RP, Falimirski ME, Holevar MR. The role of presacral drainage in the management of penetrating rectal injuries. J Trauma. 1998;45(4):656-61.

49. Velmahos GC, Gomez H, Falabella A, Demetriades D. Operative management of civilian rectal gunshot wounds: simpler is better. World J Surg. 2000;24(1):114-8.

50. Levy RD, Strauss P, Aladgem D, Degiannis E, Boffard KD, Saadia R. Extraperitoneal rectal gunshot injuries. J Trauma. 1995;38(2): 273-7.

51. Franko ER, Ivatury RR, Schwalb DM. Combined penetrating rectal and genitourinary injuries: a challenge in management. J Trauma. 1993;34(3):347-53. 
52. Kuy S, Codner PA, Guralnick M, Dua A, Paul J. Combined rectovesicular injuries from low velocity penetrating trauma in an adult. WMJ. 2013;112(1):32-4.

53. Crispen PL, Kansas BT, Pieri PG, Fisher C, Gaughan JP, Pathak AS, et al. Immediate postoperative complications of combined penetrating rectal and bladder injuries. J Trauma. 2007;62(2):325-9.

54.• Morey AF, Brandes S, Dugi 3rd DD, et al. Urotrauma: AUA guideline. J Urol. 2014;192(2):327-35. AUA guidelines on the management of traumatic $G U$ injuries.

55. Medina D, Lavery R, Ross SE, Livingston DH. Ureteral trauma: preoperative studies neither predict injury nor prevent missed injuries. J Am Coll Surg. 1998;186(6):641-4.

56. Palmer LS, Rosenbaum RR, Gershbaum MD, Kreutzer ER. Penetrating ureteral trauma at an urban trauma center: 10-year experience. Urology. 1999;54(1):34-6.
57. Morey A, Dugi H. Genital and lower urinary tract trauma. In: Wein A, Kavoussi L, Novick A, Partin A, Peters C, editors. CampbellWalsh urology. 10th ed. Philadelphia: Elsevier Saunders; 2012. p. 2507-20.

58. Ishak C, Kanth N. Bladder trauma: multidetector computed tomography cystography. Emerg Radiol. 2011;18(4):321-7.

59. Figler BD, Hoffler CE, Reisman W, et al. Multi-disciplinary update on pelvic fracture associated bladder and urethral injuries. Injury. 2012;43(8):1242-9.

60. Deibert CM, Spencer BA. The association between operative repair of bladder injury and improved survival: results from the national trauma data bank. J Urol. 2011;186(1):151-5. Large scale review of the outcomes associated with traumatic bladder injury.

61. Smith 3rd TG, Coburn M. Damage control maneuvers for urologic trauma. Urol Clin N Am. 2013;40(3):343-50. 